\title{
Cosmological Implications of Electroweak Monopole
}

\author{
Y. M. $\mathrm{Cho}^{1,2, *}$ \\ ${ }^{1}$ Administration Building 310-4, Konkuk University, Seoul 143-701, Korea \\ ${ }^{2}$ School of Physics and Astronomy, Seoul National University, Seoul 151-742, Korea
}

\begin{abstract}
In this talk we review the basic features of the electroweak monopole, and estimate the remnant electroweak monopole density of the standard model in the present universe. We show that, although the electroweak phase transition is of the first order, the monopole production comes from the thermal fluctuations of the Higgs field after the phase transition, not the vacuum bubble collisions during the phase transition. Moreover, most of the monopoles produced initially are annihilated as soon as created, and this annihilation continues very long time, longer than the muon pair annihilation time. As the result the remnant monopole density at present universe becomes very small, of $10^{-11}$ of the critical density, too small to be the dark matter. We discuss the physical implications of our results on the ongoing monopole detection experiments.
\end{abstract}

PACS numbers: $14.80 . \mathrm{Hv}, 12.15 .-\mathrm{y}, 04.20 .-\mathrm{q}$

Keywords: electroweak monopole, mass of electroweak monopole, cosmological production of electroweak monopole, remnant density of electroweak monopole, seed of large scale structure.

\section{INTRODUCTION}

Ever since Dirac has proposed the Dirac monopole generalizing the Maxwell's theory, the monopole has become an obsession in physics [1]. After the Dirac monopole we have had the Wu-Yang monopole [2], the 't Hooft-Polyakov monopole [3], the grand unification (Dokos-Tomaras) monopole [4], and the electroweak (Cho-Maison) monopole [5, 6]. But only the electroweak monopole become realistic enough to be discovered.

This has made the experimental confirmation of the electroweak monopole one of the most urgent issues in the standard model after the discovery of the Higgs particle, and the newest MoEDAL ("the magnificent seventh") detector at LHC is actively searching for the monopole $[7,8]$. On the other hand, the $13 \mathrm{TeV}$ LHC may have no chance to produce the monopole if the monopole mass becomes larger than $6.5 \mathrm{TeV}$. In this case we must try to detect the remnant monopole at present universe produced in the early universe. so it is fortunate that IceCube, Antares, Auger, and similar experiments are searching for them $[9-11]$

To detect the remnant monopoles, however, we need to know how many of them are left over in the present universe. There have been discussions on the cosmological production of monopoles, but most of them have been on the grand unification monopoles [12-15]. The general consensus is that the grand unification monopoles would have overclosed the universe without the inflation, but the inflation might have completely diluted them in such a way that they could have no visible impact on the present universe [16].

*Electronic address: ymcho7@konkuk.ac.kr
For the electroweak monopole, however, the situation was not so clear [17-19]. There have been claims that the electroweak monopoles could also overclose the universe [18], but we need a more accurate discussion on this issue. The purpose of this talk is to discuss the cosmological production and successive evolution of the electroweak monopole to predict the remnant monopole density of the standard model at present universe, and discuss the cosmological implications of the electroweak monopole.

Before we estimate the electroweak monopole density at present universe, we emphasize the basic facts on the electroweak monopole. First, it is the electroweak generalization of the Dirac monopole. But unlike the Dirac monopole which is optional, the electroweak monopole must exist because the electromagnetic $\mathrm{U}(1)$ in the standard model becomes non-trivial $[5,6]$. This makes the discovery of the monopole, not the Higgs particle, the final (and topological) test of the standard model.

Second, there is a fundamental difference between the Dirac monopole and the electroweak monopole. It carries the magnetic charge twice bigger than the Dirac monopole. This is because, in the course of the electroweak unification, the period of electromagnetic $\mathrm{U}(1)$ becomes $4 \pi$, not $2 \pi[5,6]$. So we could tell if it is the Dirac monopole or the electroweak monopole, when we discover it.

Third, unlike the Dirac monopole, we can estimate the mass of the electroweak monopole to be of the order of several TeV, roughly $1 / \alpha$ times the $\mathrm{W}$-boson mass [2022 . This is because the monopole mass essentially comes from the same Higgs mechanism which generates the Wboson mass, except that the monopole potential couples to the Higgs multiplet magnetically.

Unfortunately there have been confusions and misunderstandings on the electroweak monopole in the literature. A most serious one is the assertion that the stan- 
dard model has mo monopole because the theory has no monopole topology [23]. This "non-existence theorem", however, is a false assertion based on wrong topology. To clarify these confusions and misunderstandings, we briefly review the electroweak monopole in the standard model first.

\section{ELECTROWEAK MONOPOLE: A REVIEW}

Consider the Weinberg-Salam model,

$$
\begin{gathered}
\mathcal{L}=-\left|\mathcal{D}_{\mu} \phi\right|^{2}-\frac{\lambda}{2}\left(\phi^{\dagger} \phi-\frac{\mu^{2}}{\lambda}\right)^{2}-\frac{1}{4} \vec{F}_{\mu \nu}^{2}-\frac{1}{4} G_{\mu \nu}^{2}, \\
\mathcal{D}_{\mu} \phi=\left(\partial_{\mu}-i \frac{g}{2} \vec{\tau} \cdot \vec{A}_{\mu}-i \frac{g^{\prime}}{2} B_{\mu}\right) \phi
\end{gathered}
$$

where $\phi$ is the Higgs doublet, $\vec{A}_{\mu}$ and $B_{\mu}$ are the SU(2) and $\mathrm{U}(1)$ gauge potentials. Introducing the Higgs field $\rho$ and the $C P^{1}$ field $\xi$ by

$$
\phi=\frac{1}{\sqrt{2}} \rho \xi, \quad\left(\xi^{\dagger} \xi=1\right),
$$

we have

$$
\begin{aligned}
\mathcal{L}= & -\frac{1}{2}\left(\partial_{\mu} \rho\right)^{2}-\frac{\rho^{2}}{2}\left|\mathcal{D}_{\mu} \xi\right|^{2}-\frac{\lambda}{8}\left(\rho^{2}-\rho_{0}^{2}\right)^{2} \\
& -\frac{1}{4} \vec{F}_{\mu \nu}^{2}-\frac{1}{4} G_{\mu \nu}^{2}, \quad\left(\rho_{0}^{2}=\frac{2 \mu^{2}}{\lambda}\right) .
\end{aligned}
$$

With the ansatz

$$
\begin{gathered}
\rho=\rho(r), \quad \xi=i\left(\begin{array}{c}
\sin (\theta / 2) e^{-i \varphi} \\
-\cos (\theta / 2)
\end{array}\right), \\
\vec{A}_{\mu}=\frac{1}{g} A(r) \partial_{\mu} t \hat{r}+\frac{1}{g}(f(r)-1) \hat{r} \times \partial_{\mu} \hat{r} \\
B_{\mu}=\frac{1}{g^{\prime}} B(r) \partial_{\mu} t-\frac{1}{g^{\prime}}(1-\cos \theta) \partial_{\mu} \varphi
\end{gathered}
$$

we have the dyon solution of the standard model dressed by the W-boson, Z-boson, and Higgs field, which becomes the monopole solution with $A=B=0$. The singular monopole solution is shown in Fig. $1[5,6,20]$.

The most serious misunderstanding on the electroweak monopole is the non-existence. It has been asserted that the vacuum of the Weinberg-Salam model does not allow the monopole topology, and thus has no monopole [23]. Obviously the existence of the monopole solution assures that the standard model has the monopole $[5,6,20]$. If so, the remaining question is if this monopole is optional or not. It is not. The electroweak monopole must exist, if the standard model is correct.

To see this notice that in the ansatz (4) the hypercharge $\mathrm{U}(1)$ is made to be non-trivial, which was why the electroweak monopole could exist. So we have to find the

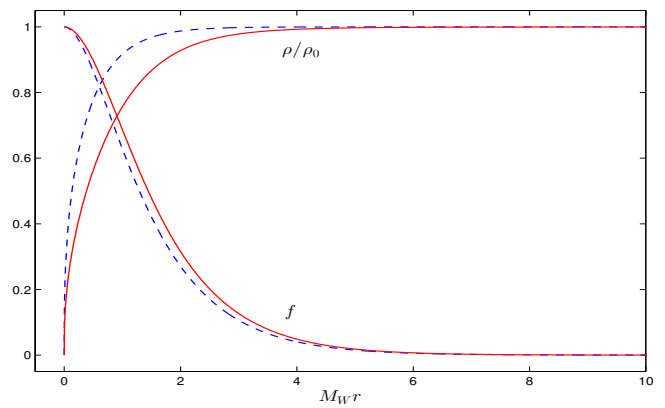

FIG. 1: The finite energy electroweak monopole solution. The solid line (red) represents the regularized monopole and the dotted (blue) line represents the singular electroweak monopole.

reason why the hypercharge $\mathrm{U}(1)$ in the standard model must be non-trivial. This follows from two facts. First, the electromagnetic $\mathrm{U}(1)$ in the standard model is given by the linear combination of the $\mathrm{U}(1)$ subgroup of $\mathrm{SU}(2)$ and the hypercharge U(1). Second, the U(1) subgroup of $\mathrm{SU}(2)$ is non-trivial. In this case the mathematical consistency requires the hypercharge $\mathrm{U}(1)$, and consequently the electromagnetic $\mathrm{U}(1)$, to be non-trivial.

But, of course, this has to be confirmed by experiment. This makes the discovery of the monopole, not the Higgs particle, the final (and topological) test of the standard model. This is why the electroweak monopole is so important.

Another important question is whether there is any characteristic feature of the electroweak monopole which is different from the Dirac monopole. The answer is yes. The electroweak monopole carries the magnetic charge twice bigger than the Dirac monopole $[5,20]$. The reason is simple. It is well known that the magnetic charge of Dirac monopole must be a multiple of $2 \pi / e$. This is because the period of the electromagnetic $\mathrm{U}(1)$ is $2 \pi$. However, in the course of the electroweak unification the period of electromagnetic $\mathrm{U}(1)$ becomes $4 \pi$, because the period of the $\mathrm{U}(1)$ subgroup of $\mathrm{SU}(2)$ becomes $4 \pi$. Consequently the magnetic charge $g_{m}$ of the electroweak monopole must be a multiple of $4 \pi / e$.

The third assertion is that we can not estimate the mass of the electroweak monopole, because the electroweak monopole has the point singularity at the origin which makes the energy infinite. Obviously the monopole mass is the most important piece of information from the experimental point of view. There was no way to predict the mass of the Dirac monopole theoretically, and this has made the monopole detection a blind search in the dark room without any theoretical lead.

But this assertion is not true, either. In fact we could easily guess the mass to be of the order of $10 \mathrm{TeV}$, roughly $4 \pi / e^{2}$ times the $\mathrm{W}$-boson mass. This is because the 
monopole mass essentially comes from the same Higgs mechanism which generates the $\mathrm{W}$-boson mass, except that the monopole potential couples to the Higgs multiplet magnetically, with the strength $4 \pi / e[5,20]$.

Another way to estimate the mass is to regularize the monopole to make the energy finite [20]. To do this let us modify (3) to the following effective Lagrangian with a non-trivial permittivity $\epsilon$ for the hypercharge $\mathrm{U}(1)$ gauge field

$$
\begin{gathered}
\mathcal{L}_{e f f}=-\frac{1}{2}\left(\partial_{\mu} \rho\right)^{2}-\frac{\rho^{2}}{2}\left|\mathcal{D}_{\mu} \xi\right|^{2}-\frac{\lambda}{8}\left(\rho^{2}-\rho_{0}^{2}\right)^{2} \\
-\frac{1}{4} \vec{F}_{\mu \nu}^{2}-\frac{\epsilon(\rho)}{4} G_{\mu \nu}^{2} .
\end{gathered}
$$

The effective Lagrangian still retains the $\mathrm{SU}(2) \times \mathrm{U}(1)$ gauge symmetry. Moreover, when $\epsilon=1$, the Lagrangian reproduces the standard model. So this modification affects only the short distance behavior, if $\epsilon$ approaches to the unit asymptotically.

To see how $\epsilon$ mimics the quantum correction and regularize the monopole, notice that the rescaling of $B_{\mu}$ to $B_{\mu} / g^{\prime}$ changes $g^{\prime}$ to $g^{\prime} / \sqrt{\epsilon}$. So $\epsilon$ effectively changes the hypercharge $\mathrm{U}(1)$ gauge coupling $g^{\prime}$ to the "running" coupling $\bar{g}^{\prime}=g^{\prime} / \sqrt{\epsilon}$. This means that, by making $\bar{g}^{\prime}$ infinite (requiring $\epsilon$ vanishing) at the origin, we can remove the singularity of the monopole. Moreover, with $\epsilon(\infty)=1$, we can reproduce the singular monopole asymptotically. In fact, with $\epsilon=\left(\rho / \rho_{0}\right)^{8}$, we have the regularized monopole solution which has the energy around $7 \mathrm{TeV}[20,22]$. This is shown in Fig. 1. Notice that asymptotically the regularized monopole looks almost identical to the singular monopole.

The regularized monopole energy, of course, depends on the functional form of $\epsilon$, so that we could change the energy changing $\epsilon$. But this also affects other things, for example the Higgs to two photon decay rate. And recently Ellis and collaborators noticed that, choosing a more realistic $\epsilon$ which can reproduce the experimental value of the Higgs to two photon decay rate, they could reduce the monopole energy less than $5.5 \mathrm{TeV}$.

\section{GRAVITATIONALLY COUPLED ELECTROWEAK MONOPOLE}

Now we discuss the gravitational modification of the monopole. Intuitively, the gravitational modification is expected to be negligible. But there is the possibility that the gravitational attraction could change the monopole to a black hole and make the monopole mass arbitrary [24]. We show that this happens only when the Higgs vacuum value approaches to the Planck scale.

Consider the effective Lagrangian (5) minimally coupled to Einstein's theory. For the sake of simplicity, we focus on the static spherically symmetric solutions and assume the space-time metric to be

$$
\begin{gathered}
d s^{2}=-N^{2}(r) A(r) d t^{2}+\frac{d r^{2}}{A(r)}+r^{2}\left(d^{2} \theta+\sin ^{2} \theta d \varphi^{2}\right), \\
A(r)=1-\frac{2 G m(r)}{r} .
\end{gathered}
$$

With the ansatz (4) the reduced Einstein-WeinbergSalam action becomes

$$
\begin{gathered}
S=\int\left[\frac{1}{4 \pi} \dot{m}-A K-U\right] N d r \\
K=\frac{\dot{f}^{2}}{g^{2}}+\frac{r^{2}}{2} \dot{\rho}^{2}, \\
U=\frac{\left(1-f^{2}\right)^{2}}{2 g^{2} r^{2}}+\frac{\lambda}{8} r^{2}\left(\rho^{2}-\rho_{0}^{2}\right)^{2}+\frac{\epsilon(\rho)}{2 g^{\prime 2} r^{2}}+\frac{1}{4} f^{2} \rho^{2},
\end{gathered}
$$

where the dot represents $d / d r$.

From this we have the following equations of motion

$$
\begin{gathered}
\frac{\dot{N}}{N}=8 \pi G \frac{K}{r}, \quad \dot{m}=4 \pi(A K+U), \\
A \ddot{f}+\left(\dot{A}+A \frac{\dot{N}}{N}\right) \dot{f}+\frac{1-f^{2}}{r^{2}} f-\frac{1}{4} g^{2} \rho^{2} f=0, \\
A \ddot{\rho}+\left(\frac{2 A}{r}+\dot{A}+A \frac{\dot{N}}{N}\right) \dot{\rho}-\frac{f}{2 r^{2}} \rho \\
-\frac{\lambda}{2}\left(\rho^{2}-\rho_{0}^{2}\right) \rho-\frac{1}{2 g^{\prime 2} r^{4}} \frac{d \epsilon(\rho)}{d \rho}=0 .
\end{gathered}
$$

This has two limiting solutions. First, when gravitational field decouples (i.e., when $G \rightarrow 0$ ) we have the nongravitating electroweak monopole solution [20]. Second, when $f=0, \rho=\rho_{0}$, and $\epsilon=1$, we have the magnetically charged Reissner-Nordstrom black hole solution with the magnetic charge [24]. But in general the solution depends on three parameters

$$
\alpha=\sqrt{G} \rho_{0}=\rho_{0} / M_{p}, \quad \beta=M_{H} / M_{W},
$$

and the Weinberg angle $\theta_{W}$.

Depending on the boundary conditions the entire solutions of (8) can be classified into two categories: the globally regular solutions and the black holes. Asymptotic flatness of the space-time requires that both $N(r)$ and $m(r)$ become constants at spatial infinity, which requires the following boundary conditions on the $\mathrm{W}$-boson and Higgs field,

$$
f(\infty)=0, \quad \rho(\infty)=\rho_{0}
$$

We fix the scale of the time coordinate adopting

$$
N(\infty)=1
$$

Notice that $m(\infty)$ which determines the total mass of the monopole is not constrained. 


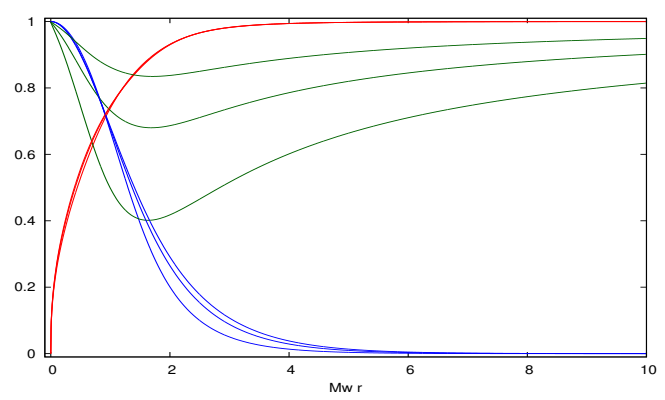

FIG. 2: The W-boson $f$ (blue), Higgs field $\rho$ (red), and the metric function $A(r)=1-2 G m / r$ (green) profiles of the gravitating monopole solutions in the standard model. The black curves represent the finite energy solution in flat spacetime, and the other three are obtained with $\alpha=\rho_{0} / M_{P}=$ $0.1,0.2$, and 0.38 .

For the regular monopole solutions we require the regularity at the origin,

$$
f(0)=1, \quad \rho(0)=0, \quad m(0)=0 .
$$

Now, choosing $\epsilon=\left(\rho / \rho_{0}\right)^{8}$ for simplicity, we find that the solutions have the following expansions near $r=0$,

$$
\begin{gathered}
f(r)=1-f_{1} x^{2}+\ldots, \\
\rho(r)=h_{1} \rho_{0} x^{\delta_{1}}+\ldots, \quad\left(\delta_{1}=\frac{\sqrt{3}-1}{2}\right) \\
m(r)=\frac{2 \pi \alpha^{2} h_{1}^{2} \delta_{1}^{2}}{G M_{W} \delta_{2}} x^{\delta_{2}}+\ldots, \quad\left(\delta_{2}=\sqrt{3}\right),
\end{gathered}
$$

where $x=M_{W} r$. From this we can obtain the solutions by the standard shooting method with $f_{1}$ and $h_{1}$ as the shooting parameters. The result shows that when $\alpha=\rho_{0} / M_{P}$ is less than 0.39 , we have a completely regular magnetically charged solution which can describe the monopole star. But when $\alpha$ approaches to 0.39, the solution turns to a magnetic blackhole. This is schematically shown in Fig. 2. Notice that, except for the metric, the gravitating monopole looks very much like the non-gravitating monopole. In particular, the size of the monopole remains roughly about $1 / M_{W}$.

To find the mass of the monopole, first note that with (8) we have

$$
\begin{gathered}
m(r)=4 \pi e^{P(r)} \int_{0}^{r}(K+U) e^{-P\left(r^{\prime}\right)} d r^{\prime}, \\
P(r)=8 \pi G \int_{r}^{\infty} \frac{K}{r^{\prime}} d r^{\prime}
\end{gathered}
$$

So, with (10) we have the total mass given by

$$
\mathcal{M}=m(\infty)=4 \pi \int_{0}^{\infty}(K+U) e^{-P(r)} d r
$$

which assures the positivity of the total mass. Moreover, this confirms that the gravitation reduces the monopole

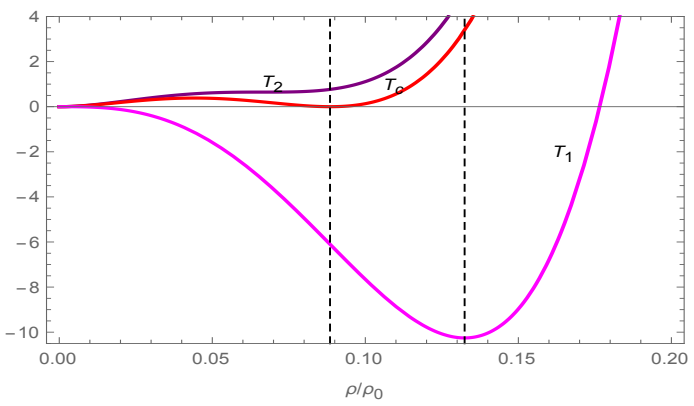

FIG. 3: The amplified effective thermal potential for $T=$ $T_{2}, T_{c}$, and $T_{1}$. Here the vertical axis represents $V_{\text {eff }}-V_{0}$ in the unit $10^{-5} \times V_{0}$, and the vertical dashed lines indicate the positions of the local minima of the potentials.

mass, which is expected from the attractive nature of the gravitational interaction. In reality, of course, the gravitational modification of the monopole mass becomes negligible because $\alpha$ is very small (of $10^{-16}$ ).

\section{ELECTROWEAK PHASE TRANSITION}

Now we discuss the electroweak phase transition. To do this, we have to find the temperature-dependent effective potential of the Higgs field. Fortunately this has been known [17, 19],

$$
\begin{gathered}
V_{T}(\rho)=V_{0}(\rho)-\frac{C_{1}}{12 \pi} \rho^{3} T+\frac{C_{2}}{2} \rho^{2} T^{2}-\frac{\pi^{2}}{90} N T^{4}+\delta V_{T} \\
V_{0}(\rho)=\frac{\lambda}{8}\left(\rho^{2}-\rho_{0}^{2}\right)^{2} \\
C_{1}=\frac{6 M_{W}^{3}+3 M_{Z}^{3}}{\rho_{0}^{3}} \simeq 0.36 \\
C_{2}=\frac{4 M_{W}^{2}+2 M_{Z}^{2}+M_{H}^{2}+4 m_{t}^{2}}{8 \rho_{0}^{2}} \simeq 0.36
\end{gathered}
$$

where $V_{0}$ is the zero-temperature potential, $N$ is the total number of distinct helicity states of the particles with mass smaller than $T$ (counting fermions with the factor $7 / 8), C_{1}$ and $C_{2}$ are the contributions from the $\rho$ dependent part of the masses of gauge bosons, Higgs field, and fermions, $M_{W}, M_{Z}, M_{H}$, and $m_{t}$ are the W-boson, Zboson, Higgs boson, and the top quark masses, and $\delta V_{T}$ is the slow-varying logarithmic corrections and the lighter quark contributions which we will neglect from now on.

The effective potential (with $\delta V_{T}=0$ ) is schematically drawn in Fig. 3. It has three local extrema at

$$
\begin{gathered}
\rho_{s}=0, \\
\rho_{ \pm}(T)=\frac{C_{1}}{4 \pi \lambda} T \pm \sqrt{\left(\frac{C_{1}}{4 \pi \lambda}\right)^{2} T^{2}+\rho_{0}^{2}-\frac{2 C_{2}}{\lambda} T^{2}} .
\end{gathered}
$$

The first extremum $\rho_{s}=0$ represents the Higgs vacuum of the symmetric (unbroken) phase, and the second extremum $\rho_{-}(T)$ represents the local maximum, and 
the third extremum $\rho_{+}(T)$ represent the local minimum Higgs vacuum of the broken phase. But notice that these two extrema $\rho_{ \pm}$appear only when $T$ becomes smaller than $T_{2}$

$$
\begin{gathered}
T_{2}=\frac{T_{1}}{\sqrt{1-C_{1}^{2} / 16 \pi^{2} \lambda C_{2}}} \simeq 146.7 \mathrm{GeV}, \\
T_{1}=\sqrt{\frac{\lambda}{2 C_{2}}} \rho_{0} \simeq 146.4 \mathrm{GeV},
\end{gathered}
$$

So above this temperature only $\rho_{s}=0$ becomes the true vacuum of the effective potential, and the electroweak symmetry remains unbroken.

At $T=T_{2}$ we have

$$
\rho_{-}=\rho_{+}=\left(C_{1} / 4 \pi \lambda\right) T_{2} \simeq 16.3 \mathrm{GeV},
$$

but as temperature cools down below $T_{2}$ we have two local minima at $\rho_{s}$ and $\rho_{+}$with $V_{T}(0)<V_{T}\left(\rho_{+}\right)$, until $T$ reaches the critical temperature $T_{c}$ where $V_{T}(0)$ becomes equal to $V_{T}\left(\rho_{+}\right)$,

$$
\begin{gathered}
T_{c}=\frac{T_{1}}{\sqrt{1-C_{1}^{2} / 18 \pi^{2} \lambda C_{2}}} \simeq 146.6 \mathrm{GeV}, \\
\rho_{+}\left(T_{c}\right)=\frac{C_{1}}{3 \pi \lambda} T_{c} \simeq 21.8 \mathrm{GeV} .
\end{gathered}
$$

So $\rho_{s}=0$ remains the minimum of the effective potential for $T>T_{c}$. Notice that $T_{c} / \rho_{0} \simeq 0.6$ but $\rho_{+} / \rho_{0} \simeq 0.09$.

Below this critical temperature $\rho_{+}$becomes the true minimum of the effective potential, but $\rho_{s}=0$ remains a local (unstable) minimum till the temperature reaches $T_{1}$. But at $T=T_{c}$ the new vacuum bubbles start to nucleate at $\rho=\rho_{+}$, which takes over the unstable vacuum $\rho_{s}=0$ completely at $T=T_{1}$ where $\rho_{+}\left(T_{1}\right)$ becomes around $32.6 \mathrm{GeV}$. From this point $\rho_{+}$becomes the only (true) minimum, which transforms to the wellknown Higgs vacuum $\rho_{0}$ at zero temperature.

This tells that two local minima $\rho_{s}$ and $\rho_{+}$are separated by an energy barrier, which means that the electroweak phase transition is of the first order. On the other hand, the energy barrier is very small. Moreover, the barrier lasts only for short period since the temperature difference from $T_{c}$ to $T_{1}$ is very small, $\delta=$ $\left(T_{c}-T_{1}\right) / T_{c} \simeq 0.001$. This strongly implies that the phase transition is very mildly the first order, almost the second order.

From (17) we can clculate the temperature-dependent Higgs mass $\bar{M}_{H}$,

$$
\begin{gathered}
\bar{M}_{H}^{2}=\left.\frac{d^{2} V_{e f f}}{d \rho^{2}}\right|_{\rho_{\min }} \\
= \begin{cases}{\left[\left(T / T_{1}\right)^{2}-1\right] M_{H}^{2} / 2,} & T \geq T_{c}, \\
\left.\left(\rho_{+} / \rho_{0}\right)^{2}+1-\left(T / T_{1}\right)^{2}\right] M_{H}^{2} / 2, & T<T_{c} .\end{cases}
\end{gathered}
$$

So $\bar{M}_{H}$ acquires its minimum value $5.53 \mathrm{GeV}$ at $T=T_{c}$ and becomes $11.7 \mathrm{GeV}$ at $T=T_{1}$, and approaches to the

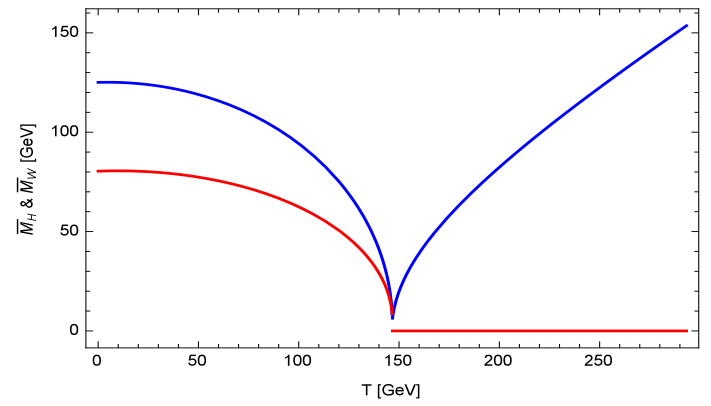

FIG. 4: The temperature-dependent Higgs and W-boson masses. The blue and red curves represent the Higgs and W-boson masses.

zero temperature value $125.7 \mathrm{GeV}$ as the universe cools down.

Similarly, the W-boson which was massless at high temperature becomes massive at $T=T_{c}$ when the Higgs field acquires the non-vanishing vacuum expectation value. So, as the Higgs vacuum $\rho=0$ starts to tunnel to $\rho=\rho_{+}$at $T_{c}$, the $\mathrm{W}$-boson starts to become massive toward the value $g \rho_{+}\left(T_{c}\right) / 2 \simeq 7.0 \mathrm{GeV}$. And it acquires the mass $10.5 \mathrm{GeV}$ at $T=T_{1}$, which approaches the well-known zero-temperature value at $T=0$. The temperature-dependent Higgs and W-boson masses are shown in Fig. 4. They play an important role because they determine the correlation length $\xi=\bar{M}_{H}^{-1}$ and the monopole mass $M_{m} \simeq(1 / \alpha) \times \bar{M}_{W}$.

The cosmological production of monopole in the second order phase transition was estimated by Kibble and later improved by Zurek [12,15]. Kibble observed that the fundamental scale which determines the initial monopole density in the second order phase transition is the horizon distance at the time of the transition, and set a upper bound for the initial monopole density. Zurek improved this bound taking into account the fact that the phase transition does not take place instantaneously but continuously.

But this picture does not apply to the electroweak monopole production, because the electroweak phase transition is the first order. In this case, the correlation length becomes finite because the Higgs mass becomes non-zero at the critical temperature. Moreover, what is important in the monopole production is the time of monopole formation. To produce the monopoles we need the change of topology induced by the zero points of the Higgs field, which comes from the thermal fluctuations. And obviously these zero points, the seeds of the monopoles, do not appear instantaneously at the critical temperature, but come to exist some time after the phase transition. And it is well known that the temperature which governs this thermal fluctuation is the Ginzburg temperature, not the critical temperature. This means that even in the second order phase transition, the relevant scale in the monopole production is not the horizon 
distance at the critical temperature but the correlation length around the Ginzburg temperature.

Of course, in the strongly first order phase transition, the symmetric vacuum becomes meta-stable below the critical temperature, and the vacuum bubbles nucleate during the phase transition. So the vacuum bubble collisions during the phase transition becomes the monopole production mechanism [14]. However, the electroweak phase transition is very mildly first order, so that the bubble formation becomes unimportant. So the monopole formation takes place by the thermal fluctuation of the Higgs field after the phase transition, not by the bubble collisions during the phase transition.

Just below the critical temperature the Higgs field is still subject to large fluctuations which bring $\langle\rho\rangle$ back to zero. This is possible so long as

$$
\xi^{3} \Delta F \leq T
$$

where $\xi(T)$ is the correlation length and $\Delta F(T)=$ $V\left(\rho_{s}\right)-V\left(\rho_{+}\right)$is the difference in free energy density between two phases. This large fluctuation disappears when the equality holds, at the Ginzburg temperature. So we have to find the Ginzburg temperature first.

Unfortunately the Ginzburg temperature $T_{G}$ of the potential (17) can not be expressed in a simple form, but we can find $T_{G}$ numerically directly from (17) and (22),

$$
T_{G} \simeq 57.6 \mathrm{GeV}, \quad \xi_{G} \simeq 1.7 \times 10^{-16} \mathrm{~cm} .
$$

With this we may assume that the period of the monopole formation is between $T_{1}$ and $T_{G}$, and identify the temperature and correlation length of the initial monopole production to be the mean value of $T_{1}$ and $T_{G}$,

$$
\begin{gathered}
T_{i}=\frac{T_{1}+T_{G}}{2} \simeq 102.0 \mathrm{GeV} \\
\xi_{i}=\frac{\xi\left(T_{1}\right)+\xi\left(T_{G}\right)}{2} \simeq 9.3 \times 10^{-16} \mathrm{~cm}
\end{gathered}
$$

From this we can estimate the initial density of the monopoles

$$
\left(\frac{n_{m}}{T^{3}}\right)_{i} \simeq \frac{g_{P}}{\xi_{i}^{3} T_{i}^{3}} \simeq 9.1 \times 10^{-4}
$$

where $g_{P}$ is the probability that one monopole is produced in one correlation volume, which we assume to be around $10 \%$.

To see how much energy we need to produce the electroweak monopoles, we calculate the energy density of the monopoles from (26)

$$
\begin{gathered}
\varrho_{m o}\left(T_{i}\right)=M_{m}\left(n_{m}\right)_{i} \simeq 9.0 \times 10^{-3} T_{i}^{4}\left(\frac{M_{m}}{1 \mathrm{TeV}}\right), \\
\frac{\varrho_{m o}\left(T_{i}\right)}{\varrho\left(T_{i}\right)} \simeq 2.5 \times 10^{-4}\left(\frac{M_{m}}{1 \mathrm{TeV}}\right) .
\end{gathered}
$$

So, assuming the initial monopole mass to be around 1 $\mathrm{TeV}$, we may conclude that the universe need to consume only a tiny fraction (about $0.025 \%$ ) of the total energy to produce the monopoles. This assures that the electroweak monopole production does not alter the standard cosmology.

In fact, we can say that even (26) is an overestimation. The reason is that, as we will see soon, the monopoleantimonopole capture radius becomes much bigger the correlation length $\xi_{i}$, so that most of the monopoles annihilate with the anti-monopoles as soon as they are produced.

\section{COSMIC EVOLUTION OF ELECTROWEAK MONOPOLE}

Although the monopoles are topological and absolutely stable, there are two factors which change the initial monopole density, the Hubble expansion and the annihilation of monopole-antimonopole pairs. So the change of monopole density $n_{m}$ is determined by the Boltzmann equation $[12,13]$

$$
\frac{d n_{m}}{d t}+3 H n_{m}=-\sigma n_{m}^{2} .
$$

where $H$ and $\sigma$ are the Hubble parameter and the monopole annihilation cross section.

There are two things which affect the annihilation of the monopoles, the thermal Brownian motion (random walk) of the monopole in hot plasma of charged particles and the attraction between monopoles and antimonopoles. After the creation the monopoles diffuse in hot plasma by the Brownian motion with the mean free path $l_{\text {free }}$ given by

$$
l_{\text {free }}=v_{t} t_{\text {free }} \simeq \frac{1}{B T} \sqrt{\frac{M_{m}}{T}}, \quad B=\frac{1}{T} \sum_{i} n_{i} \sigma_{i},
$$

where $v_{t} \simeq \sqrt{T / M_{m}}$ and $t_{\text {free }}$ are the thermal velocity and the mean free time of the monopoles, $n_{i}$ and $\sigma_{i}$ are the number density and the cross section of the $i$-th relativistic charged particles and the sum is the sum over all spin states [13].

With

$$
n_{i} \simeq \frac{3 \zeta(3)}{4 \pi^{2}} T^{3}, \quad \sigma_{i} \simeq\left(\frac{g_{m} q_{i}}{4 \pi}\right)^{2} \frac{1}{T^{2}}=\left(\frac{q_{i}}{e}\right)^{2} \frac{1}{T^{2}},
$$

we have

$$
B \simeq \frac{3 \zeta(3)}{4 \pi^{2}} \sum_{i}\left(\frac{q_{i}}{e}\right)^{2} \simeq 0.09 \times \sum_{i}\left(\frac{q_{i}}{e}\right)^{2},
$$

where $q_{i}$ is the electric charge of the $i$-th particle and $\zeta(3)=1.202 \ldots$ is the Riemann zeta function. Since 


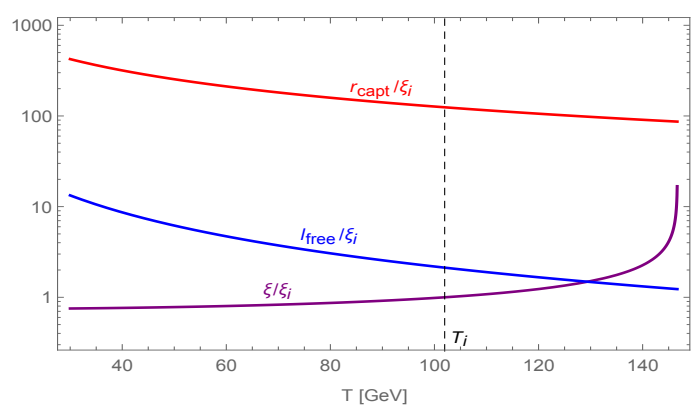

FIG. 5: The relevant scales, $\xi$ in purple, $l_{\text {free }}$ in blue, and $r_{\text {capt }}$ in red, against $T$. They are normalized by the correlation length $\xi_{i}$ at $T_{i}$. Here we set $M_{m}=5 \mathrm{TeV}$.

the charged particles in the plasma are the leptons and quarks, we may put $B \simeq 3$. From this we have $v_{t} \simeq 0.31 \times$ $\left(1 \mathrm{TeV} / M_{m}\right)^{1 / 2} c$ and $l_{\text {free }} \simeq 2.0 \times\left(M_{m} / 1 \mathrm{TeV}\right)^{1 / 2} \times$ $10^{-16} \mathrm{~cm}$ around $T_{i}$.

Now, against the thermal random walk of the monopoles, we have the attractive Coulomb force between monopoles and anti-monopoles which makes them drift towards each other. The monopole drift velocity $v_{d}$ at a distance $r$ from the anti-monopole is given by

$$
v_{d} \simeq \frac{1}{\alpha} \times \frac{1}{B T^{2} r^{2}}=\frac{\alpha_{m}}{B T^{2} r^{2}},
$$

where $\alpha_{m}$ is the monopole fine structure constant defined by $\alpha_{m}=g_{m}^{2} / 4 \pi=1 / \alpha$. Notice that $v_{d}\left(r=\xi_{i}\right) \simeq 2 c$. This, of course, is unrealistic but tells that $v_{t} \ll v_{d}$. This shows that the Coulomb attraction between the monopoles and anti-monopoles is much stronger than the thermal diffusion.

Moreover, the monopole-antimonopole capture radius given by $r_{\text {capt }} \simeq \alpha_{m} / T$ is much bigger than the mean free path $l_{\text {free. }}$. This strongly implies that the monopole-antimonopole annihilation is much more important around $T_{i}$, so that as soon as they are created, they annihilate each other. In Fig. 5 we plot the relevant scales $\xi, l_{\text {free }}$, and $r_{\text {capt }}$ against $T$ for comparison. Notice that the capture radius is much bigger than the mean free length and correlation length. This assures that (26) is indeed an overestimation.

Now, if we let the mean distance between the monopole and anti-monopole $r$ be $r \simeq n_{m}^{-1 / 3}$, the capture time is given by

$$
t_{\text {capt }} \simeq \frac{r}{v_{d}} \simeq \alpha \times \frac{B T^{2}}{n_{m}} .
$$

From this we have the monopole-antimonopole annihilation cross section

$$
\sigma \simeq \frac{1}{t_{\text {capt }} n_{m}} \simeq \frac{\alpha_{m}}{B T^{2}}
$$

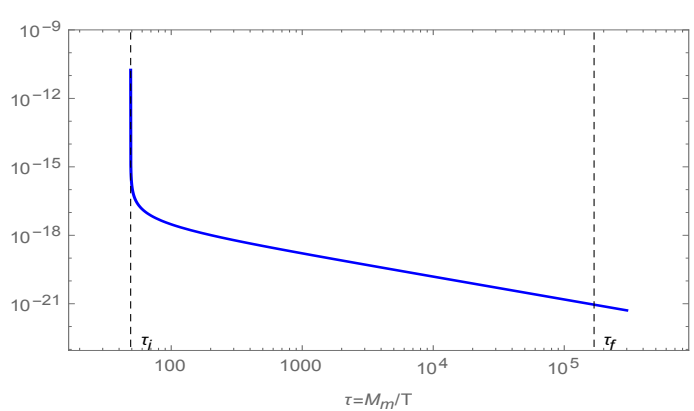

FIG. 6: The evolution of the monopole density $n_{M} / T^{3}$ against $\tau=M_{m} / T$. The final value of the monopole density is independent of the initial value.

With this we can solve the Boltzmann equation (28),

$$
\begin{gathered}
\frac{n_{M}}{T^{3}}=\frac{1}{A\left(\tau-\tau_{i}\right)+B}, \\
A=\alpha_{m} \times \frac{C m_{p}}{B M_{m}}, \quad B=\left(\frac{n_{M}}{T^{3}}\right)_{i}^{-1} .
\end{gathered}
$$

Here $M_{m}$ is treated as a constant. But in reality it depends on time, so that it should be understood as a mean value.

The evolution of the monopole density $n_{m} / T^{3}$ against $M_{m} / T$ is shown in Fig. 6, where we have put $M_{m}=$ $5 \mathrm{TeV}$. Notice that, regardless of the initial condition the monopole density approaches to a constant as the universe is cooled down.

The diffusive capture process is effective only when $\ell_{\text {free }}<r_{\text {capt }}$, which determines the temperature $T_{f}$ below which the monopole-antimonopole annihilation ceases,

$$
T_{f} \simeq \alpha^{2} \times \frac{M_{m}}{B^{2}} \simeq 5.9 \times\left(\frac{M_{m}}{1 \mathrm{TeV}}\right) \mathrm{MeV},
$$

so that

$$
\tau_{f}=\frac{M_{m}}{T_{f}} \simeq\left(\alpha_{m} B\right)^{2} \simeq 1.7 \times 10^{5} .
$$

With $M_{m}=5 \mathrm{TeV}$, we have $T_{f} \simeq 29.5 \mathrm{MeV}$, which is below the muon decoupling temperature. Actually, around this temperature $B$ becomes very small so that the capture radius becomes smaller. This is because the only charged particles remaining in the plasma are electrons and positrons. But the above analysis clearly shows that the annihilation continues very long time.

The monopole density after annihilation becomes

$$
\left(\frac{n_{m}}{T^{3}}\right)_{f} \simeq \alpha^{3} \times \frac{M_{m}}{B C m_{p}} \simeq 1.8 \times 10^{-22}\left(\frac{M_{m}}{1 \mathrm{TeV}}\right) .
$$

Obviously this value is much lower than the initial density given by (26). The number of monopole within the comoving volume is conserved thereafter. But they still interact with the electron pairs in the hot plasma before 
decouple around $T_{d} \simeq 0.5 \mathrm{MeV}$, when the electron pairs disappear and the interaction rate becomes less than the Hubble expansion rate.

Assuming that the expansion is adiabatic, the current number density and the energy density of the monopole is given by

$$
\begin{gathered}
\left(\frac{n_{m}}{T^{3}}\right)_{0}=\left(\frac{g_{s, 0}}{g_{s, f}}\right)\left(\frac{n_{m}}{T^{3}}\right)_{f}, \\
\rho_{m o, 0}=M_{m} n_{m, 0}=M_{m}\left(\frac{g_{s, 0}}{g_{s, f}}\right)\left(\frac{n_{m}}{T^{3}}\right)_{f} T_{0}^{3},
\end{gathered}
$$

where $T_{f}$ is the temperature of universe when the monopole annihilation process effectively ceases and $T_{0}=$ $2.73 \mathrm{~K}=2.35 \times 10^{-13} \mathrm{GeV}$ is the temperature of the universe today. Here $g_{s}$ is the effective number of degrees of freedom in entropy.

So the current density parameter of monopole can be written

$$
\Omega_{m o} h^{2}=\frac{\rho_{m o, 0}}{\rho_{\mathrm{c}, 0} h^{-2}} \simeq 1.2 \times 10^{-12} \times\left(\frac{M_{m}}{1 \mathrm{TeV}}\right)^{2},
$$

where $\rho_{\mathrm{c}, 0}=3 H_{0}^{2} / 8 \pi G$ is the critical density of present universe and $h \simeq 0.678$ is the scaled Hubble constant in the unit $H_{0} /\left(100 \mathrm{~km} \mathrm{~s}^{-1} \mathrm{Mpc}^{-1}\right)$. With $h \simeq 0.678$ and $M_{m} \simeq 5 \mathrm{TeV}$, we have $\Omega_{m o} \simeq 6.53 \times 10^{-11}$. This is about $1.31 \times 10^{-9}$ of the baryon density, which assures that the electroweak monopole cannot be a dark matter candidate.

In terms of the number density, this translates to about $6.1 \times 10^{-20} \mathrm{~cm}^{-3}$, or about $2.3 \times 10^{-13} n_{b}$, where $n_{b} \simeq 2.5 \times 10^{-7} \mathrm{~cm}^{-3}$ is the number density of the baryons. Intuitively, this means that there are roughly $6.6 \times 10^{7}$ monopoles per unit volume of the earth. This is a small but significant number which suggests that there are enough electroweak monopoles left over in the universe that we could detect.

\section{PARKER BOUND}

Obviously the monopoles are accelerated in magnetic fields, in particular the intergalactic magnetic field. It is well known that the average strength of the galactic magnetic field $B$ is about $3 \mu G \simeq 1.2 \times 10^{-9} \mathrm{~T}$. The energy gained by the monopole of charge $g_{m}=4 \pi / e$ passing across the magnetic field $B$ of scale $L$ is

$$
\Delta E=\frac{4 \pi}{e} L B \simeq 1.2 \times 10^{11} \times\left(\frac{L}{10^{21} \mathrm{~cm}}\right) \mathrm{GeV},
$$

where $L$ is normalized to the typical coherence length of galactic magnetic field $L_{0}=300 \mathrm{pc} \simeq 10^{21} \mathrm{~cm}$. Traveling through the distance $L_{0}$, the monopole drains energy $\Delta E \simeq 10^{11} \mathrm{GeV}$ from the magnetic field and becomes ultra-relativistic [25]. So, although the monopoles when decoupled around 29.5 MeV were non-relativistic, the remnant monopoles at present universe should be treated as relativistic.

Requiring that the rate of this energy loss in the galaxy is small compared to the time scale on which the galactic magnetic field can be regenerated, we can obtain the upper bound on the flux of the monopoles (with mass $\left.M_{m} \leq 10^{17} \mathrm{GeV}\right)$,

$$
F \leq 10^{-15} \mathrm{~cm}^{-2} \mathrm{sec}^{-1} \mathrm{sr}^{-1} .
$$

This is the Parker bound [26].

The Parker bound sets a limit on the monopole density in the universe. The monopoles with velocity $v_{m}$ uniformly distributed throughout the universe generate the monopole flux

$$
\begin{aligned}
F \simeq & \frac{v_{m}}{4 \pi} n_{m, 0}=\frac{v_{m}}{4 \pi}\left(\frac{\rho_{c, 0}}{h^{2} M_{m}}\right) \Omega_{m o} h^{2} \\
\simeq & 2.3 \times 10^{-2} \Omega_{m o} h^{2}\left(\frac{v_{m}}{10^{-3} c}\right) \\
& \times\left(\frac{1 \mathrm{TeV}}{M_{m}}\right) \mathrm{cm}^{-2} \mathrm{sec}^{-1} \mathrm{sr}^{-1},
\end{aligned}
$$

where $v_{m}$ is expressed in terms of the average virial velocity of the galaxy $10^{-3} c$. This, with (42), requires

$$
\Omega_{m o} h^{2} \lesssim 4.3 \times 10^{-14}\left(\frac{10^{-3} c}{v_{m}}\right) \times\left(\frac{M_{m}}{1 \mathrm{TeV}}\right) .
$$

This set a stringent limit on the density for the relativistic $\left(v_{m} \simeq c=1\right)$ electroweak monopoles. This causes us a serious trouble, because our estimate of the density parameter (40) is roughly $10^{5}$ times bigger than this limit.

We could think of possible ways to circumvent this trouble. First of all, we might suppose that the limit (44) is not trustable. Obviously this is an approximation. For example, the monopoles in the galactic magnetic field, when accelerated near the velocity of light, will make the Chrenkov radiation and will slow down to a limiting velocity considerably less than the velocity of light.

But a more realistic explanation could be that most of the electroweak monopoles in the universe are actually buried inside the galactic cores. This is quite possible, because the electroweak monopoles become the natural source for the premodial black holes and the structure formation. Certainly, as the heaviest stable particles, they could easily cause the density pertubation and become the seeds for the large scale structures in the universe.

Another possibility is that many of the electroweak monopoles are captured inside stellar objects when they hit (large) stellar objects, because they have a large capture cross section due to the magnetic interaction. In fact a relativistic electroweak monopole with mass $5 \mathrm{TeV}$ is expected to travel less than $10 \mathrm{~m}$ in Aluminum before they are trapped [27]. So the electroweak monopoles 
coming to the earth loose most of the energy passing through the earth atmospheric sphere and become nonrelativistic, and could easily be trapped near the earth surface. In fact we may conjecture that these trapped monopoles (and buried in large scale structures) could have been the very source of the intergalactic magnetic field.

More importantly, this strongly suggests that the stellar objects could have filtered out and diluted the density of the monopoles in the universe greatly, so that the remnant monopole density at present universe might have become much smaller than (26). So at this moment it is not clear whether our result is in contradiction with the Parker bound.

\section{DISCUSSIONS}

Our analysis confirms that, although the electroweak phase transition is of the first order, it is very mildly first order. So the monopole production mechanism is not the vacuum bubble collisions during the phase transition but the thermal fluctuations of the Higgs field after the phase transition, which plant the seed of the monopoles.

Our result shows that the electroweak monopoles are produced when the temperature of the universe was around $100 \mathrm{GeV}$ (or about $10^{-11} \mathrm{sec}$ after the big bang). And initially the monopoles are produced copiously, perhaps a bit too copiously to be realistic. But most of them are annihilated as soon as created, and this annihilation continues for a long time until the universe cools down to around $39.5 \mathrm{MeV}$, even after the muon pair annihilation. And eventually they decouple from the other matters at around $0.5 \mathrm{MeV}$, when the electron pairs annihilate and the monopole interaction rate becomes less than the Hubble expansion rate.

Because of this the electroweak monopole density become very small, $\Omega_{m o} \simeq 6.3 \times 10^{-11}$, in the present universe. This tells that, unlike the grand unification monopole, the electroweak monopole can not overclose the universe. As importantly, this assures that the production of the electroweak monopole does not alter the standard cosmology in any significant way, and exclude the possibility the electroweak monopole to be a candidate of the dark matter.

On the other hand, this means that there are enough monopoles left over, roughly $6.6 \times 10^{7}$ monopoles per every unit volume of the earth in the present universe. This strongly indicates that experimentally there are enough remnant monopoles that we should be able to detect without much difficulty.

However, we should to keep in mind the possibility that the actual density of the remnant monopoles could be much less than this. This is because many of them could have been buried in the the large scale structures

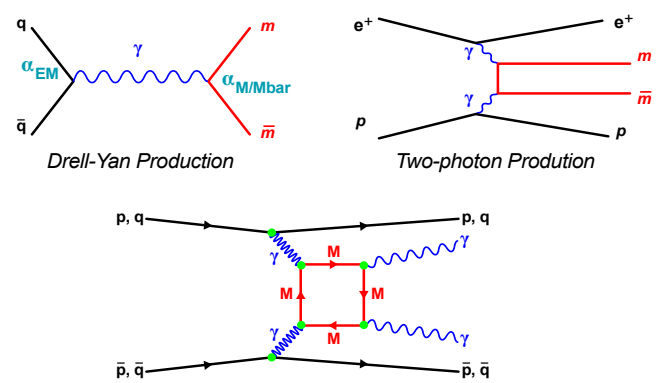

FIG. 7: The conventional electroweak monopole production mechanism. This should be compared with our topologcal production of the electroweak monopole.

of the universe and/or filtered out by stellar objects. In fact, the actual monopole density could be smaller by the factor $10^{-5}$, or about $10^{3}$ per every unit volume of the earth, as the Parker bound indicates.

A major concern at $\mathrm{LHC}$ is if it can produce the monopole. It has generally been believed that LHC could produce the monopole pair by Drell-Yan process $(p \bar{p} \rightarrow \gamma \rightarrow M \bar{M})$ and/or photon fusion process $(p \bar{p} \rightarrow$ $p \bar{p}+\gamma \gamma \rightarrow p \bar{p}+M \bar{M})$. This is shown in Fig. 7, which should be compared with our topological production mechanism. This popular view would be acceptable if we treat the monopole as a point particle. But since the monopole is a topological particle, it is not clear if this is correct.

Obviously for LHC to produce the monopole the monopole mass must be less than $6.5 \mathrm{TeV}$. In our monopole production mechanism, however, LHC should satisfy two more conditions to produce the monopole. First, it should generate the hot plasma of the beam core of the size at least as big as the initial correlation length $\xi_{i} \simeq 10^{-15} \mathrm{~cm}$. Second, this beam core should last at least ten times longer than $10^{-26}$ sec to allow enough fluctuations for the Higgs vacuum to plant the monopole seed.

Fortunately, the size of the beam core at LHC has been measured to be about $0.03 \mathrm{~cm}$ and lasts for about $10^{-12}$ sec, long enough for the Higgs field to allow more than enough thermal flucuations. This strongly implies that there is a good chance that LHC could actually produce one monopole-antimonopole pair, which MoEDAL could detect.

For the other experiments searching for the remnant monopoles in the universe, in particular for IceCube, ANTARES, and Auger, an important thing is to know the characteristic features of the remnant monopoles [911]. Although the electroweak monopoles were nonrelativistic when they decoupled, the intergalactic magnetic field makes them highly relativistic at present universe. On the other hand, they loose most of the energy passing through the earth atmospheric sphere, so that near the earth surface they become non-relativistic 
again. So on earth these experiments should look for the non-relativistic monopole coming from the sky, which has mass around 4 to $10 \mathrm{TeV}$ and magnetic charge $4 \pi / e$.

In principle these experiments have the ability to detect the monopoles, but there is one catch here. These detectors (except Auger) are located underground. This could cause a problem, because the non-relativistic remnant monopoles may be trapped before they reach the detector. For example, IceCube is located under the 2 $\mathrm{km}$ thick iceberg, which the remnant monopoles may not be able to penetrate. If so, it would be very difficult for IceCube to detect the monopole. In fact this could be a main reason why IceCube could not find it. So these experiments have to find a way to circumbent this problem to detect the remnant monopoles.

Although the remnant electroweak monopoles in the present universe are insignificant, they have important physical implications. As we have speculated, they could have been the seed of the large scale structures in the universe. Indeed the electroweak monopoles with mass about $10^{4}$ times heavier than the proton, could easily generate the density perturbation and become an excellent candidate for the seed of the large scale structures in the universe. Moreover, as the heaviest relativistic magnetically charged particles in the universe, they be- come the source of ultra-high energy cosmic rays. Furthermore, they could play an important role in the electroweak baryogenesis.

But the most important point of the electroweak monopole is that it must exist. This makes the detection of the electroweak monopole the final (topological) test of the standard model. In spite of huge efforts, however, the monopole detection so far has not been successful. There could be two reasons for this. First, many of these experiments were the blind seraches in the dark room, with few theoretical leads. Second, many were looking for different type of monopoles, in particular the grand unification monopole.

So, aiming at the electroweak monopole which has the unique characteristic features, we could enhance the probability to detect the monopole greatly. We hope that our analysis in this paper could help confirm the existence of the electroweak monopole.

\section{ACKNOWLEDGMENT}

The work is supported in part by the National Research Foundation of Korea funded by the Ministry of Education (Grants 2015-R1D1A1A0-1057578) and by Konkuk University.
[1] P.A.M. Dirac, Proc. Roy. Soc. London, A133, 60 (1931); Phys. Rev. 74, 817 (1948).

[2] T.T. Wu and C.N. Yang, Phys. Rev. D12, 3845 (1975); Y.M. Cho, Phys. Rev. Lett. 44, 1115 (1980); Phys. Lett. B115, 125 (1982).

[3] G. 't Hooft, Nucl. Phys. B79, 276 (1974); A.M. Polyakov, JETP Lett. 20, 194 (1974).

[4] C. Dokos and T. Tomaras, Phys. Rev. D21, 2940 (1980).

[5] Y.M. Cho and D. Maison, Phys. Lett. B391, 360 (1997).

[6] Yisong Yang, Proc. Roy. Soc. London, A454, 155 (1998); Yisong Yang, Solitons in Field Theory and Nonlinear Analysis (Springer Monographs in Mathematics), p. 322 (Springer-Verlag) 2001.

[7] B. Acharya et al. (MoEDAL Collaboration), Phys. Rev. Lett. 118, 061801 (2017).

[8] B. Acharya et al. (MoEDAL Collaboration), Int. J. Mod. Phys. A29, 1430050 (2014); JHEP 1608, 067 (2016); Y.M. Cho and J. Pinfold, Snowmass White paper, arXiv: [hep-ph] 1307.8390.

[9] R. Abbasi et al. (IceCube Collaboration) Phys. Rev. D87, 022001 (2013); M. Aartsen et al. (IceCube Collaboration), Eur. Phys. J. C74, 2938 (2014).

[10] S. Adrián-Martinez et al. (ANTARES Collaboration), Astropart. Phys. 35, 634 (2012).

[11] A. Aab et al. (Pierre Auger Collaboration) Phys. Rev. D94, 082002 (2016).

[12] T.W.B. Kibble, J. Phys. A9 1387 (1976).

[13] J. P. Preskill, Phys. Rev. Lett. 43, 1365 (1979).

[14] A. H. Guth and E. J. Weinberg, Nucl. Phys. B212, 321 (1983).

[15] W. H. Zurek, Phys. Rep. 276177 (1996).

[16] A. H. Guth, Phys. Rev. D23, 347 (1981); A. D. Linde,
Phys. Lett. B108 389 (1982).

[17] D.A. Krizhnits and A.D. Linde, Phys. Lett. B42, 471 (1972); C. Bernard, Phys. Rev. D9, 3312 (1974); L. Dolan and R. Jakiw, Phys. Rev. D9, 3320 (1974); S. Weinberg, Phys. Rev. D9, 3357 (1974).

[18] Y. B. Zel'dovich and M.Yu. Khlopov, Phys. Lett. 79B, 239 (1978); P.J. Adams,V. Canuto, and H.Y. Chiu, Phys. Lett. 61B, 397 (1976).

[19] G. Anderson and L. Hall, Phys. Rev. D45, 2685 (1992); M. Dine, R. Leigh, P Huet, A. Linde, and D. Linde, Phys. Rev. D46, 550 (1992).

[20] Kyoungtae Kimm, J.H. Yoon, and Y.M. Cho, Eur. Phys. J. C75, 67 (2015); Kyoungtae Kimm, J.H. Yoon, S.H. Oh, and Y.M. Cho, Mod. Phys. Lett. A31, 1650053 (2016).

[21] J. Ellis, N.E. Mavromatos, and T. You, Phys. Lett B756, 29, (2016).

[22] Y.M. Cho, Kyoungtae Kimm, J.H. Yoon, Phys. Lett. B761, 203 (2016)

[23] S. Coleman, Aspects of Symmetry (Cambridge Univ. Press, 1985); T. Vachaspati and M. Barriola, Phys. Rev. Lett. 69, 1867 (1992).

[24] F.A. Bais and R.J. Russell, Phys. Rev. D11, 2692 (1975); Y.M. Cho and P.G.O. Freund, Phys. Rev. D12, 1588 (1975).

[25] S. Burdin, M. Fairbaim, P. Mermod, D. Milstead, J. Pinfold, T. Sloan, and W. Taylor, Phys. Rep. 582, 1 (2015).

[26] E.N. Parker, Astrophys. J. 160, 383 (1970).

[27] S. Cecchini, L. Patrizii, Z. Sahnoun, G. Sirri, and V. Togo, arXiv: hep-ph/1606.01220. 